\title{
Rotation of Early B-type Stars in the Large Magellanic Cloud: The Role of Evolution and Metallicity
}

\author{
Stefan C. Keller ${ }^{\mathrm{A}, \mathrm{B}}$ \\ A Research School of Astronomy and Astrophysics, Australian National University, \\ Weston ACT 2611, Australia \\ B E-mail: stefan@mso.anu.edu.au
}

Received 2004 February 24, accepted 2004 May 18

\begin{abstract}
I present measurements of the projected rotational velocities of a sample of 100 early B-type mainsequence stars in the Large Magellanic Cloud (LMC). This is the first extragalactic study of the distribution of stellar rotational velocities. The sample is drawn from two sources: from the vicinity of the main-sequence turnoff of young clusters (ages $1-3 \times 10^{7} \mathrm{yr}$ ) and from the general field. I find that the cluster population exhibits significantly more rapid rotation than that seen in the field. I have drawn analogous Galactic cluster and field samples from the literature. Comparison of these samples reveals the same effect. I propose that the observed difference between cluster and field populations can be explained by a scenario of evolutionary enhancement of the surface angular momentum over the main-sequence lifetime. A comparison is made between the cluster and field populations of the LMC and the Galaxy in order to explore the effects of metallicity. This shows that the stars of the LMC are more rapid rotators than their Galactic counterparts.
\end{abstract}

Keywords: stars: evolution — stars: rotation — Magellanic Clouds

\section{Introduction}

This paper aims to establish the distribution of projected rotational velocities $(v \sin i)$ for a sample of early B-type stars in the Large Magellanic Cloud (LMC). I explore the effects of age and metallicity on the observed distribution of rotational velocities. The motivation for this study arises from recent stellar evolutionary models that incorporate stellar rotation. This modelling shows that the rotational history is vital to the interpretation of the evolutionary phase and elemental abundances seen in massive stars. Heger, Langer, \& Woosley (2000) and Meynet \& Maeder (2000) show that axial rotation can bring about, in a natural way, the degree of extension to the convective core in massive stars as discerned from cluster Hertzsprung-Russell (HR) diagrams (e.g. Keller, Bessell, \& Da Costa 2000, 2001a) and the mass-luminosity relation of Cepheids (Keller \& Wood 2002).

Models incorporating rotation also predict mixing of internally processed material to the surface during mainsequence (MS) evolution (the faster the initial rotation, the greater the degree of mixing). Standard evolution models (e.g. Bressan et al. 1993), on the other hand, do not. In the Magellanic Clouds (MCs), nitrogen has been shown to be over-abundant in many supergiants of spectral types B to K (e.g. Venn 1999; Lennon et al. 1996) and numerous MS B-stars (Rolleston et al. 1996; Korn et al. 2000, 2002) which is indicative of mixing of CNO-cycled material. The dispersion in $\mathrm{N}$ abundance is significantly larger than that seen in the Galaxy (McErlean, Lennon, \& Dufton 1999; Venn 1995; Gies \& Lambert 1992). This has been tentatively attributed to the presence of a greater number of rapid rotators within the lower-metallicity environs of the MCs.

At present, direct observational studies of the dependence of stellar rotation on metallicity are limited. Within the Galaxy the work of Burki \& Maeder (1977) examined the variation of mean $v \sin i$ of early B-stars with galactocentric distance, but the range of the latter was only several $\mathrm{kpc}$, which — given the galactic metallicity gradient is insufficient to draw firm conclusions regarding the role of metallicity. Recently Royer et al. (2003) have revisited this work, extending it to a broader sample of Galactic clusters with inconclusive results.

Indirect observational evidence for more rapid rotation of massive stars in metal-poor sites, like the MCs, is derived from examination of the population of Be-stars. Be-stars show Balmer emission arising from a circumstellar disk of material surrounding a rapidly rotating stellar photosphere. They form a population with which to trace stellar rotation (assuming that the onset of the Be phenomenon itself is not governed by metallicity, but rather the underlying distribution of rotational velocities). Maeder et al. (1999) examine the fraction of Be-stars, i.e. $N_{\mathrm{Be}} /\left(N_{\mathrm{B}}+N_{\mathrm{Be}}\right)$, compared to metallicity, for a sample of clusters with relevant ages $\left(1-3 \times 10^{7} \mathrm{yr}\right.$, corresponding to the maximum occurrence of Be-stars). Maeder et al. find a strong anti-correlation between the Be fraction and metallicity. This leads the authors to conclude that rotation rates are higher amongst metalpoor stars. 
The role of age in determining the resultant distribution of $v \sin i$ was first suggested by Abt \& Hunter (1962) in their study of the brighter members of the Trapezium and Pleiades clusters. This was elaborated upon by Wolff, Edwards, \& Preston (1982) and Guthrie (1984) in their studies, which contrasted the distribution of rotational velocities seen in young clusters, older associations, and field stars. The distribution of rotational velocities in the older associations and the field share a high proportion of very slow rotators compared with the young cluster sample.

Keller, Wood, \& Bessell (1999) and Keller et al. (2000, 2001b) have used the Be-star population to explore the age dependence of the rotational-velocity distribution. We have observed that the Be fraction amongst the young cluster population rises towards the luminosity of the MS terminus, whereas in the field it retains a relatively uniform distribution in luminosity. This difference between cluster and field populations can, we argue (Keller et al. 2001b), be brought about from an evolutionary enhancement in the rotational velocity over the later portion of the MS lifetime.

It is the aim of this paper to present direct measurements of the projected rotational velocities for a sample of early B-type MS stars within young cluster and field environments of the LMC, together with analogous Galactic data from the literature, to investigate directly the dependence of $v \sin i$ on both age and metallicity. Section 2 presents our observations and method for the determination of projected rotational velocities. Section 3.1 compares the distribution of rotational velocities in the cluster and field environments of the Galaxy and the LMC. Section 3.2 compares the Galactic and LMC cluster populations to explore metallicity effects. Finally, in Section 4, I discuss the physical causes of the observed dependence of projected rotational velocities on age and metallicity.

\section{Observations and Data Reduction}

I have defined two sample sets:

1. A cluster sample drawn from the population in the vicinity of three young populous clusters in the LMC, namely, NGC 1818, NGC 2004, and NGC 2100. Our targets were selected from the WFPC2-based photometry of Keller et al. (2000). On the basis of this photometry I have selected those stars on the MS with $14<V<16$.

2. A field sample from the vicinity of NGC 2004 and NGC 1818. These regions were selected to form the basis of our sample because of their richness and apparently small differential reddening (Keller et al. 1999).

Medium resolution $\left(0.6 \AA \mathrm{px}^{-1}\right)$ spectra of the sample were obtained using the Double-Beam Spectrograph (DBS) on the Siding Spring Observatory (SSO) 2.3-m telescope from 1999 March 4-6, 1999 November 1625 , and 2001 January 10-12. The spectra consist of two simultaneously recorded non-overlapping segments: blue
(3800-4800 $\AA$ ) and red (6200-6800 $)$. The observations made use of aperture plates machined to locate the target stars. In general the density of the targets precluded the use of slits for each object, rather holes of appropriate size for typical seeing at the site ( 2 arcsec) were made. Sky correction was later made through the use of slits at the extremities of the field. Wavelength calibration was made through $\mathrm{CuAr}$ spectra interleaved with the observations.

\subsection{Determination of Rotational Velocities}

To determine $v \sin i$, I used a $\chi^{2}$-minimisation technique, which locates an optimal match between the observed spectrum and rotationally broadened synthetic spectra. The grid of synthetic profiles of stellar Balmer and He I lines by González, Delgado, \& Leitherer (1999) was used. This grid of models spans $50000 \geq T_{\text {eff }} \geq 4000 \mathrm{~K}$ and $0 \geq \log g \geq 5$. For $T_{\text {eff }}>25000 \mathrm{~K}$ NLTE models were used to compute the synthetic spectra; for cooler stars Kurucz LTE models were used.

To create the rotationally broadened spectra the synthetic spectra were first convolved with the instrumental response function and then with a broadening function, which is based upon that of Gray (1976). The model stellar surface is divided into 40 segments and the emergent Doppler-shifted intensity from each is calculated. This is then integrated to yield the flux at each wavelength.

This approach assumes that the resultant line profile is independent of viewing angle. Physically, the surface gravity (and hence temperature) of a rotating star varies with latitude as a result of gravity darkening (von Zeipel 1924). As a consequence, the contribution of the rapidly rotating cooler equatorial regions to the observed spectrum is reduced. Townsend et al. (2004) present a survey of the behaviour of line width with $v \sin i$, including viewingangle-dependent gravity darkening. For instance, for a star rotating at $95 \%$ of the critical velocity (i.e. the velocity at which the equatorial escape velocity drops to zero) and seen pole-on, using the method we apply here leads to an underestimation of $v_{\mathrm{e}} \sin i$ by $20 \%$. The underestimation of $v_{\mathrm{e}} \sin i$ is a strong function of $v_{\mathrm{e}}$, such that by $v_{\mathrm{e}}=300 \mathrm{~km} \mathrm{~s}^{-1}$, for a B2 star, this has fallen to a few percent. The neglect of gravity darkening underlies all rotational velocity standards and previous studies of rotational velocity distributions. For this reason the present work maintains this simplification.

I have used a wavelength range of 3990 to $4550 \AA$ for the purposes of finding an optimal match. The spectrum is fit as one contiguous piece. An optimal solution is sought in $v \sin i, T_{\mathrm{eff}}$, and $\log g$ parameter space. The spectra of a series of rotational velocity standards (Slettebak et al. 1975) of early- to mid-B spectral type were obtained. Figure 1 shows the comparison between the $v \sin i$ determined by means of our parameter fit procedure and that of Slettebak et al. Figure 2 shows a typical fit to the rotational velocity standards. Figure 1 does not show any systematic difference between these results and those of Slettebak et al. 
The signal-to-noise ratio $(\mathrm{S} / \mathrm{N})$ of the spectra of our LMC sample is low; a typical $3-\mathrm{hr}$ exposure results in $\mathrm{S} / \mathrm{N}=30$ for a $V=15.5$ star. On the basis of simulations (discussed below), I set a lower limit to our sample of

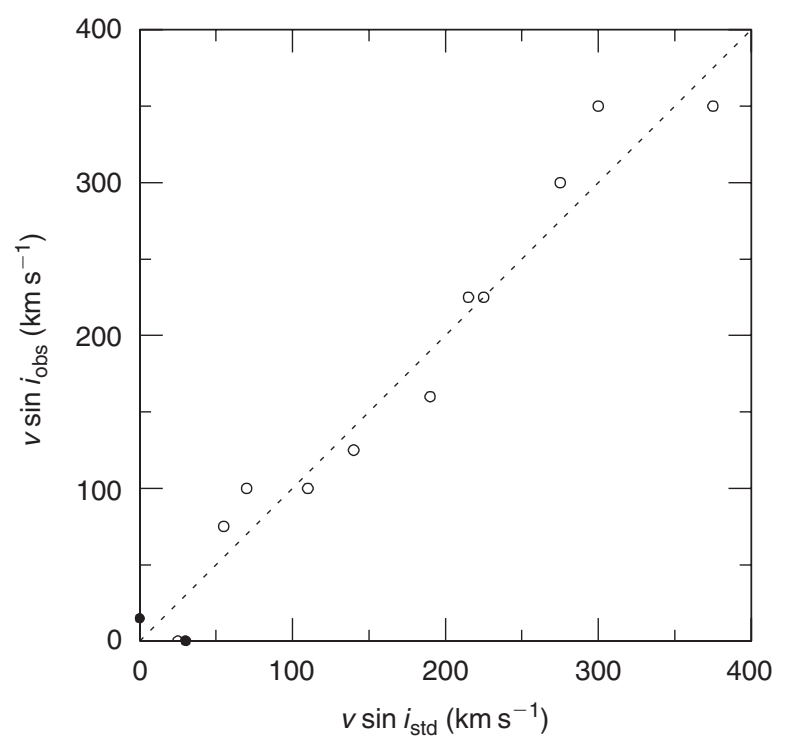

Figure 1 Comparison between projected rotational velocities of Slettebak et al. (1975, open circles) and those of the present study. Solid points show the positions of B15 and B30 in NGC 2004, from Korn et al. (2000).
$\mathrm{S} / \mathrm{N}=20(V \sim 16)$. Tables 1 and 2 report the $v \sin i$ for our sample. Some typical fits are shown in Figure 3.

The uncertainties in our estimated $v \sin i$ are a function of both $\mathrm{S} / \mathrm{N}$ and $v \sin i$. I carried out a series of trials using model spectra which are rotationally broadened and then degraded to a given $\mathrm{S} / \mathrm{N}$. I then examined the $v \sin i$ returned by our routine from repeated trials. Slow rotators are perturbed by a low $\mathrm{S} / \mathrm{N}$. A S/N of 20 introduces an uncertainty of $\pm 50 \mathrm{~km} \mathrm{~s}^{-1}$ at $v \sin i=0$ and systematically returns a higher $v \sin i$. This overestimation of $v \sin i$ at low $\mathrm{S} / \mathrm{N}$ results primarily from the radial-velocity correction, which must be made for each star during fitting. At low $\mathrm{S} / \mathrm{N}$ the centres of the significant $\mathrm{H}$ and $\mathrm{He}$ lines are less distinct and an optimal fit may occur with either a slightly higher or lower radial velocity than the underlying systemic radial velocity. A S/N-degraded line seen off-centre appears as a broader line and this results in the systematic shift of the slowest rotators to higher $v \sin i$.

As $v \sin i$ is increased, the importance of this effect diminishes. At $v \sin i=100 \mathrm{~km} \mathrm{~s}^{-1}$ and $\mathrm{S} / \mathrm{N}=20$ the uncertainty in $v \sin i$ falls to $\pm 25 \mathrm{~km} \mathrm{~s}^{-1}$, with no significant systematic shift. The uncertainty grows again to $\pm 75 \mathrm{~km} \mathrm{~s}^{-1}$ at $v \sin i=350 \mathrm{~km} \mathrm{~s}^{-1}(\mathrm{~S} / \mathrm{N}=20)$, as the line depth is reduced, but again there is no significant systematic shift. This behaviour is summarised in Figure 4. By describing in detail the systematic behaviour of our technique at low $\mathrm{S} / \mathrm{N}$ it is possible to remove it statistically, as described in Section 3.2. a. $\zeta \mathrm{Cru}$
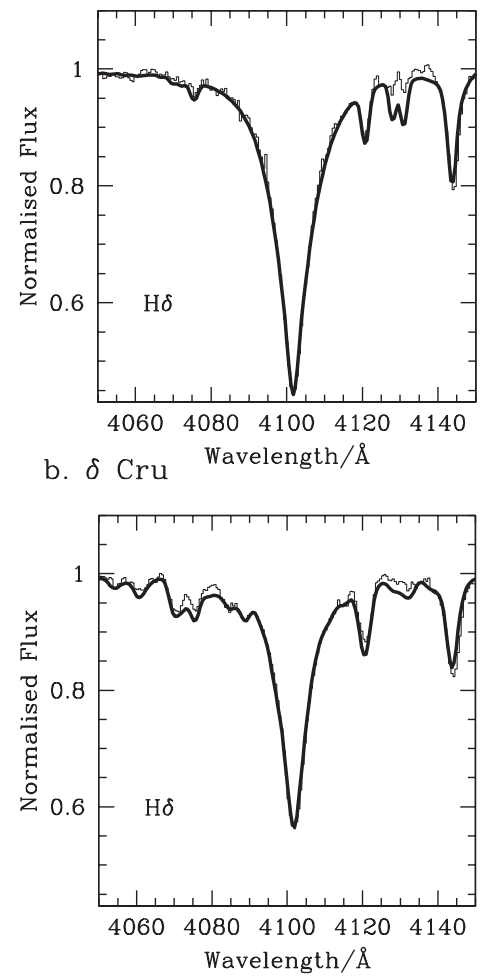
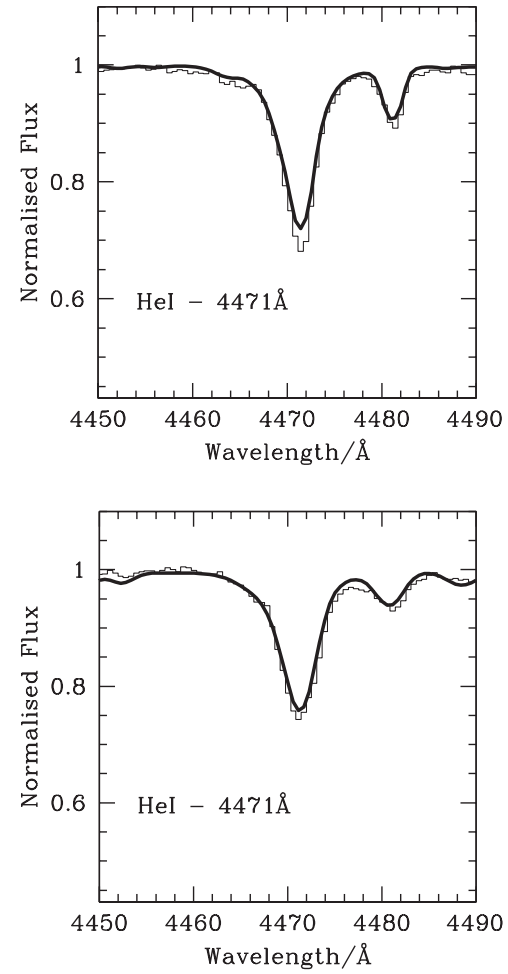

Figure 2 Examples of the fits between artificially broadened theoretical profiles (thick line) and observed stellar spectra for rotational velocity standards: a) $\zeta$ Cru $\left(\mathrm{B} 2.5 \mathrm{~V}, v \sin i=70 \mathrm{~km} \mathrm{~s}^{-1}\right)$ and b) $\delta$ Cru (B2 IV, $v \sin i=140 \mathrm{~km} \mathrm{~s}^{-1}$ ). 
Table 1. $v \sin i$ for the sample of MS stars drawn from the LMC clusters NGC 1818, 2004 and 2100. $V$ magnitudes are taken from Robertson (1974). $v \sin i$ with an additional colon indicate those objects for which the determination of $v \sin i$ has considerable uncertainty $\left( \pm 75 \mathrm{~km} \mathrm{~s}^{-1}\right)$

\begin{tabular}{lcclcr}
\hline Star & $\begin{array}{c}V \\
{[\mathrm{mag}]}\end{array}$ & $\begin{array}{c}v \sin i \\
{\left[\mathrm{~km} \mathrm{~s}^{-1}\right]}\end{array}$ & Star & $\begin{array}{c}V \\
{[\mathrm{mag}]}\end{array}$ & $\begin{array}{r}v \sin i \\
{\left[\mathrm{~km} \mathrm{~s}^{-1}\right]}\end{array}$ \\
\hline NGC2004:C10 & 15.90 & 190 & NGC1818:B34 & 14.27 & 125 \\
NGC2004:C9 & 15.76 & 150 & NGC1818:A31 & 14.89 & 0 \\
NGC2004:C8 & 14.88 & 130 & NGC1818:C24 & 16.21 & $38:$ \\
NGC2004:B15 & 14.18 & 15 & NGC1818:B39 & 15.89 & 260 \\
NGC2004:B12 & 15.31 & 187 & NGC1818:C25 & 15.74 & $0:$ \\
NGC2004:B18 & 14.77 & 190 & NGC1818:C29 & 15.59 & 0 \\
NGC2004:B38 & 14.06 & 300 & NGC1818:C27 & 15.97 & 260 \\
NGC2004:D10 & 15.11 & 150 & NGC1818:D12 & 13.74 & 0 \\
NGC2004:D8 & 15.37 & 263 & NGC1818:C11 & 16.10 & $300:$ \\
NGC2004:D3 & 15.77 & 130 & NGC1818:B24 & 15.05 & 260 \\
NGC2004:D15 & 15.10 & 105 & NGC1818:D6 & 15.57 & 180 \\
NGC2004:D13 & 13.36 & 150 & NGC1818:D4 & 15.83 & $300:$ \\
NGC2004:D16 & 13.66 & 225 & NGC2100:D30 & 14.29 & 260 \\
NGC2004:B9 & 13.48 & 0 & NGC2100:D28 & 14.91 & 120 \\
NGC2004:B24 & 14.68 & 130 & NGC2100:D31 & 14.89 & 225 \\
NGC2004:C13 & 14.90 & $265:$ & NGC2100:D26 & 15.29 & 187 \\
NGC2004:B30 & 13.83 & 15 & NGC2100:C9 & 13.78 & 15 \\
NGC2004:B28 & 15.25 & 300 & NGC2100:B45 & 14.25 & 0 \\
NGC2004:C16 & 14.69 & 105 & NGC2100:B20 & 13.71 & 120 \\
NGC2004:D7 & 15.04 & 280 & NGC2100:C30 & 14.93 & 90 \\
NGC2004:D1 & 12.02 & 15 & NGC2100:C13 & 13.85 & 105 \\
NGC1818:C10 & 16.12 & 188 & NGC2100:C15 & 13.68 & 30 \\
NGC1818:D15 & 16.21 & $150:$ & NGC2100:C27 & 15.09 & 225 \\
NGC1818:B28 & 16.13 & $225:$ & NGC2100:C20 & 13.56 & 0 \\
NGC1818:B29 & 15.67 & 225 & & & \\
\hline & & & & &
\end{tabular}

Table 2. $\quad v \sin i$ for the sample of MS B-stars drawn from the LMC field. $V$ photometry is that of the author

\begin{tabular}{|c|c|c|c|c|c|c|c|c|c|}
\hline Star & $\begin{array}{c}\text { RA } \\
{[\mathrm{J} 2000]}\end{array}$ & $\begin{array}{c}\text { Dec } \\
{[\mathrm{J} 2000]}\end{array}$ & $\begin{array}{c}V \\
{[\mathrm{mag}]}\end{array}$ & $\begin{array}{c}v \sin i \\
{\left[\mathrm{~km} \mathrm{~s}^{-1}\right]}\end{array}$ & Star & $\begin{array}{c}\text { RA } \\
{[\mathrm{J} 2000]}\end{array}$ & $\begin{array}{c}\text { Dec } \\
{[\mathrm{J} 2000]}\end{array}$ & $\begin{array}{c}V \\
{[\mathrm{mag}]}\end{array}$ & $\begin{array}{c}v \sin i \\
{\left[\mathrm{~km} \mathrm{~s}^{-1}\right]}\end{array}$ \\
\hline fp1_1 & 05:34:10.07 & $-67: 03: 12.5$ & 14.99 & 225 & fp2_13 & $05: 32: 53.80$ & $-66: 57: 20.1$ & 14.62 & 75 \\
\hline fp1_2 & $05: 33: 48.80$ & $-67: 02: 03.2$ & 15.97 & 180: & fp2_14 & $05: 32: 44.15$ & $-66: 56: 36.3$ & 14.83 & 75 \\
\hline fp1_3 & 05:34:03.63 & $-67: 01: 50.5$ & 14.69 & 100 & fp3_1 & $05: 28: 24.39$ & $-68: 15: 21.8$ & 15.53 & 90 \\
\hline fp1_4 & 05:33:55.94 & $-67: 04: 29.2$ & 15.74 & 0 & fp3_2 & $05: 29: 04.88$ & $-68: 13: 46.5$ & 14.02 & 130 \\
\hline fp1_5 & 05:34:18.87 & $-67: 03: 20.0$ & 15.73 & 175 & fp3_3 & $05: 29: 36.93$ & $-68: 02: 52.7$ & 15.19 & 240 \\
\hline fp1_6 & 05:34:01.92 & $-67: 02: 41.2$ & 15.92 & $0:$ & fp3_4 & $05: 29: 43.25$ & $-68: 19: 04.2$ & 15.42 & 150 \\
\hline fp1_7 & $05: 34: 06.28$ & $-67: 02: 35.5$ & 15.12 & 100 & fp3_5 & $05: 29: 57.43$ & $-68: 10: 38.3$ & 15.90 & 15 \\
\hline fp1_8 & 05:33:53.33 & $-67: 02: 00.2$ & 15.92 & 175 & fp3_6 & $05: 30: 12.32$ & $-68: 18: 32.0$ & 14.09 & 50 \\
\hline fp1_9 & 05:33:46.18 & $-67: 01: 30.0$ & 15.62 & 260 & fp3_7 & 05:30:40.69 & $-68: 02: 52.5$ & 15.88 & 75 \\
\hline fp1_10 & 05:33:39.99 & $-67: 01: 14.4$ & 15.85 & 340 & fp3_8 & $05: 30: 44.98$ & $-68: 06: 23.9$ & 15.89 & 20 \\
\hline fp1_11 & 05:34:01.48 & $-67: 00: 40.0$ & 14.86 & 110 & fp3_9 & $05: 30: 58.87$ & $-68: 15: 39.5$ & 15.86 & 50 \\
\hline fp1_12 & $05: 33: 59.25$ & $-67: 00: 33.1$ & 14.91 & 125 & fp3_10 & 05:31:00.15 & $-68: 09: 03.2$ & 15.33 & 20 \\
\hline fp1_13 & 05:34:05.85 & $-67: 00: 18.5$ & 15.98 & 190 & fp3_11 & $05: 31: 32.10$ & $-68: 18: 56.6$ & 15.99 & 250 \\
\hline fp1_14 & 05:34:00.17 & $-66: 59: 57.0$ & 15.77 & 225 & fp3_12 & 05:31:37.05 & $-68: 10: 59.5$ & 15.99 & 0 \\
\hline fp2_1 & $05: 32: 57.32$ & $-67: 00: 35.8$ & 15.89 & 190 & fp3_13 & $05: 31: 49.94$ & $-68: 08: 43.7$ & 15.46 & 260 \\
\hline fp2_2 & 05:33:09.98 & $-67: 00: 27.7$ & 15.71 & 260 & fp4_1 & $05: 28: 26.30$ & $-68: 12: 26.9$ & 15.44 & 0 \\
\hline fp2_3 & 05:33:16.88 & $-67: 00: 20.2$ & 15.06 & 0 & fp4_2 & $05: 28: 37.01$ & $-68: 10: 41.4$ & 15.93 & 180 \\
\hline fp2_4 & 05:33:04.07 & $-66: 59: 35.1$ & 13.99 & 0 & fp4_3 & $05: 29: 41.34$ & $-68: 22: 16.2$ & 15.96 & 0 \\
\hline fp2_5 & $05: 32: 41.76$ & $-66: 59: 17.8$ & 15.63 & 225 & fp4_4 & $05: 30: 18.30$ & $-68: 19: 08.3$ & 15.61 & 0 \\
\hline fp2_6 & 05:32:50.91 & $-66: 59: 13.8$ & 15.71 & 0 & fp4_5 & $05: 30: 40.49$ & $-68: 00: 30.6$ & 15.77 & 20 \\
\hline fp2_7 & $05: 32: 33.72$ & $-66: 59: 06.2$ & 14.91 & 110 & fp4_6 & $05: 31: 18.35$ & $-68: 10: 50.7$ & 15.18 & 0 \\
\hline fp2_8 & $05: 32: 53.60$ & $-66: 59: 02.2$ & 13.82 & 190 & fp4_7 & $05: 31: 32.87$ & $-68: 02: 23.8$ & 15.03 & 80 \\
\hline fp2_9 & 05:32:40.64 & $-66: 58: 25.5$ & 15.98 & $0:$ & fp4_8 & $05: 31: 48.81$ & $-68: 10: 33.0$ & 15.10 & 300 \\
\hline fp2_10 & $05: 32: 43.23$ & $-66: 58: 13.6$ & 15.93 & 110: & fp4_9 & $05: 32: 10.98$ & $-68: 08: 15.9$ & 15.92 & 60 \\
\hline fp2_11 & 05:33:16.46 & $-66: 57: 58.1$ & 15.69 & 260 & fp4_10 & $05: 32: 13.38$ & $-68: 15: 30.7$ & 15.36 & 0 \\
\hline fp2_12 & $05: 32: 46.78$ & $-66: 57: 42.6$ & 15.01 & 0 & & & & & \\
\hline
\end{tabular}


a. $\mathrm{fp} 2 \_8$

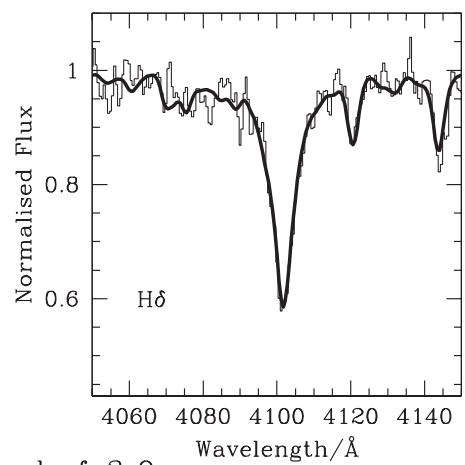

b. fp3_9

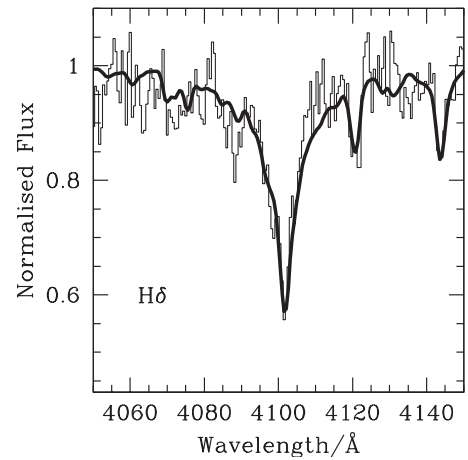

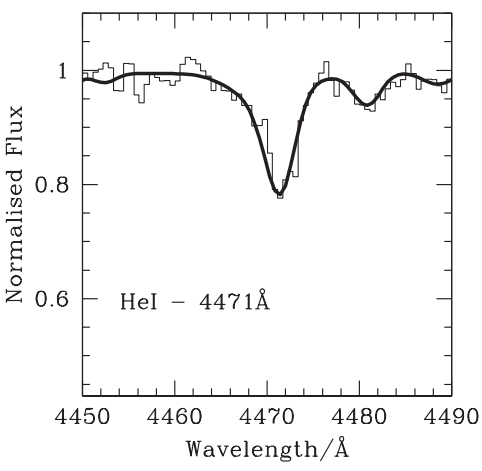

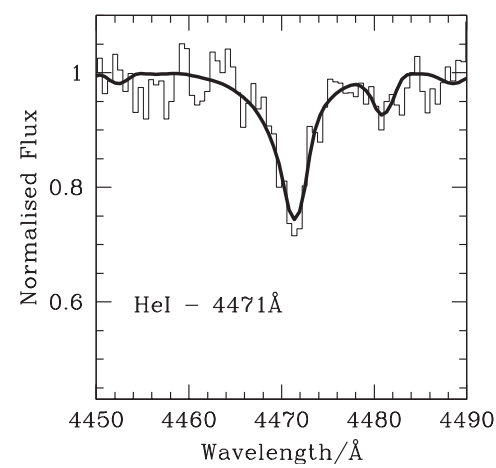

Figure 3 Examples of rotationally broadened line profile fits for LMC field stars. a) shows fp2_8 $\left(V=13.83\right.$ and $\left.v \sin i=190 \mathrm{~km} \mathrm{~s}^{-1}\right)$ and b) shows fp3_9 $\left(V=15.89\right.$ and $\left.v \sin i=50 \mathrm{~km} \mathrm{~s}^{-1}\right)$.

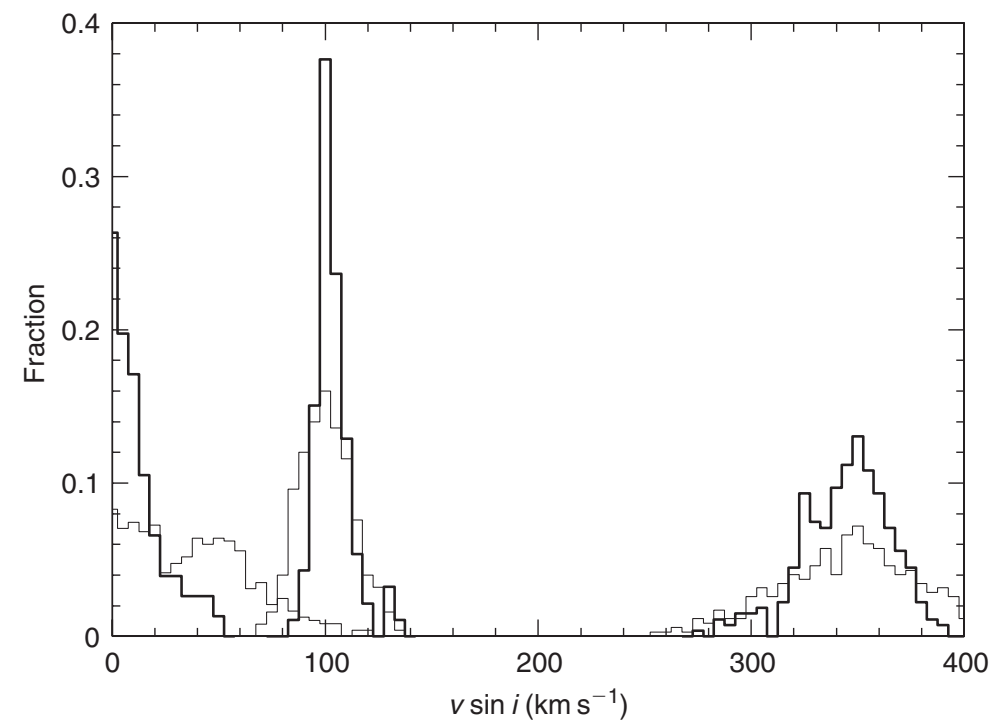

Figure 4 Histograms of the $v \sin i$ returned from the fitting routine from three models with different input $v \sin i\left(0,100\right.$ and $\left.350 \mathrm{~km} \mathrm{~s}^{-1}\right)$ and $\mathrm{S} / \mathrm{N}$. The thick line shows the distribution recovered from $\mathrm{S} / \mathrm{N}=50$ and the thinner line from $\mathrm{S} / \mathrm{N}=20$.

\section{Distribution of Rotational Velocities}

\subsection{Comparison of Cluster and Field Populations}

Figure 5 compares the distribution of rotational velocities from the LMC cluster and field samples. The nonparametric, two-sample Kolmogorov-Smirnov (KS) test is conducted to test the validity of the proposition that both samples are derived from the same parent distribution.
Such a test reveals that the cluster and field are unlikely to arise from a similar parent distribution at $2 \sigma$ significance $\left(\operatorname{Prob}\left(D>D^{+}\right)=4 \% ; D^{+}=0.25, N_{1}=49, N_{2}=51\right)$.

To extend the analysis to a discussion of the role of metallicity in the distribution of rotational velocity, I have constructed an analogous sample from the Galactic field. Our LMC sample has a limit of $V \sim 16$ imposed by the 


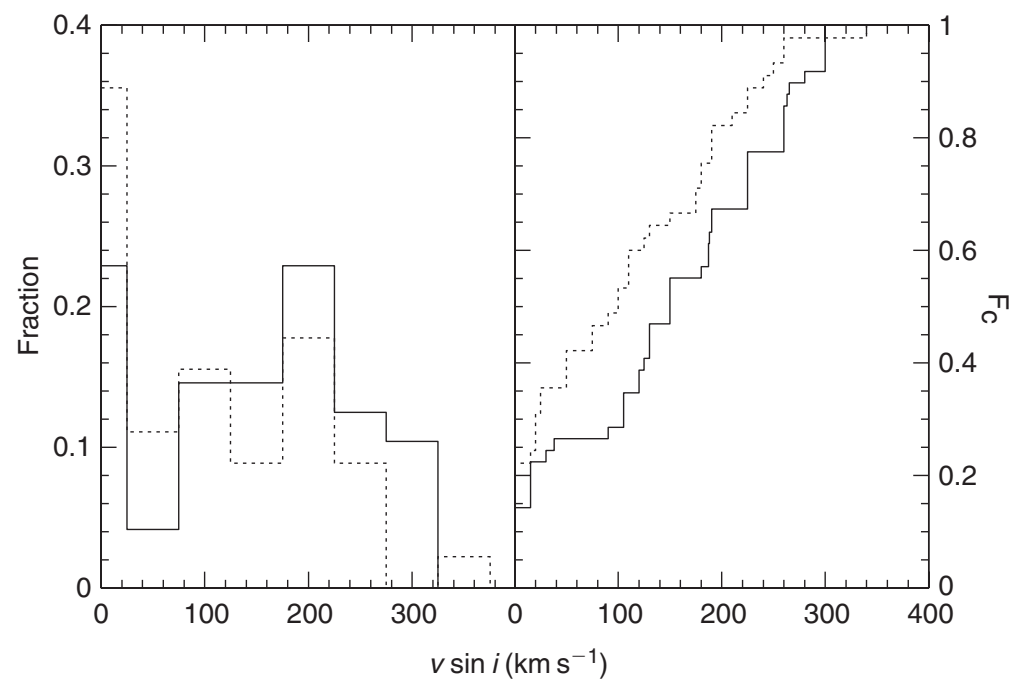

Figure 5 The distribution of rotational velocities of the 49 LMC cluster stars (solid line) and 51 field stars (dotted line), shown on the left as a histogram and on the right as the non-parametric cumulative distribution.

attainable $\mathrm{S} / \mathrm{N}$. Due to the high inclination angle and the essentially disk-like morphology of the LMC, we can regard the sample as effectively volume-limited. Assuming a distance modulus to the LMC of 18.45 our sample extends to $M_{\mathrm{V}}=-3$. This corresponds to a spectral type of B2 on the MS (Zorec \& Briot 1991).

The Galactic field sample is drawn from the Bright Star Catalogue (Hoffeit \& Jaschele 1982, hereafter BSC) and the Supplement to the Bright Star Catalogue (Hoffeit, Saladyga, \& Wlasuk 1988, hereafter SBSC). I have drawn from the catalogue stars of spectral types B0-B2 and of luminosity class III-V. The BSC+SBSC sample is magnitude-limited at $V=7.1$. In order to compare with the volume-limited sample from the LMC, we first must reduce the apparent number of stars within each spectral type and luminosity class division to a volumelimited distribution. This must be done otherwise the most luminous stars (i.e. early spectral type and high luminosity class) will receive a disproportionate weighting (Malmquist bias).

I shall consider a common volume defined by the B0 III stars. The sample-filling factor, $\epsilon$, for each spectral type and luminosity class is given in Zorec \& Briot (1997, their table 4) for the BSC+SBSC sample. The corrected histogram distribution of $v \sin i, h_{\text {cor }}(v \sin i)$, is constructed from the sum of the histograms for the individual spectral types and luminosity classes, $C$, scaled by $\epsilon$ :

$$
h_{\mathrm{cor}}(v \sin i)=\sum_{i} \epsilon_{i} h\left(C_{i}: v \sin i\right),
$$

where $\epsilon$ ranges from 4.5 for $\mathrm{B} 2 \mathrm{~V}$ to 1.0 for B0 III. The net result is a distribution which is strongly weighted by the input of the intrinsically most numerous members, that is, those of B2 V.

A distribution of projected rotational velocities was drawn from a sample of young Galactic clusters (see Table 3). The chosen clusters are rich clusters with analogous ages to those of the LMC cluster sample. Stars within
Table 3. Input to the Galactic cluster $v \sin i$ distribution

\begin{tabular}{|c|c|c|c|}
\hline Cluster & $\log ($ age/yr) & $\begin{array}{l}\text { Number of } \\
\text { stars with } \\
\text { measured } \\
v \sin i\end{array}$ & Reference \\
\hline$h+\chi$ Per. & 7.0 & 30 & Slettebak (1968) \\
\hline NGC3766 & 7.4 & 10 & Bernacca \& Perinotto (1971) \\
\hline NGC2439 & 7.1 & 17 & Bernacca \& Perinotto (1971) \\
\hline IC 4665 & 7.5 & 18 & Mermilliod (2000) \\
\hline Sco. OB2 & 7.1 & 46 & Brown \& Verschueren (1997) \\
\hline NGC 663 & 7.2 & 30 & Bernacca \& Perinotto (1971) \\
\hline
\end{tabular}

2 mag of the MS turnoff (B0-2 V-III) were selected for the distribution.

The distributions of rotational velocities for the two samples are compared in Figure 6. The results of a KS test on the above distributions shows that the Galactic cluster stars are significantly faster rotators than their field counterparts $\left(\operatorname{Prob}\left(D>D^{+}\right)=2 \%, D^{+}=0.165, N_{1}=310\right.$, $\left.N_{2}=151\right)$.

\subsection{Comparison of Galactic and LMC Populations}

Let us now compare the distributions of $v \sin i$ in both the LMC and Galactic samples, to investigate the possible role of metallicity in determining $v \sin i$. Figure 7 shows the cumulative distribution functions of the cluster and Galactic field samples (solid and dotted lines respectively). As discussed in Section 2.1, the accuracy of our determination of $v \sin i$ is critically limited by the obtainable S/N. To account statistically for the systematic effects imposed by low $\mathrm{S} / \mathrm{N}$, I took the spectral type $v \sin i$ data of the Galactic field sample and produced a set of synthetic spectra with a distribution of $\mathrm{S} / \mathrm{N}$ mimicking that of the LMC sample. I then determined the $v \sin i$ distribution of the population, as for the LMC sample. This is shown in Figure 7 as the dashed line. As expected, the net effect is to shift a proportion of the slowest rotators to higher $v \sin i$. 


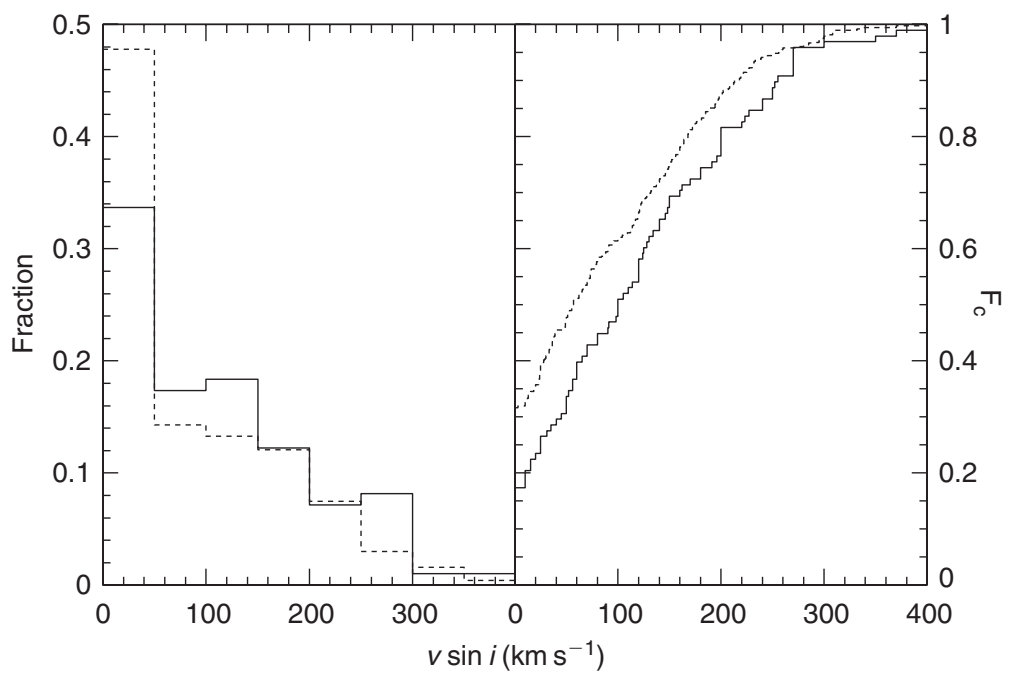

Figure 6 The distribution of rotational velocities from the Galactic cluster (solid line) and field (dotted line) shown on the left as a histogram and on the right as the non-parametric cumulative distribution.

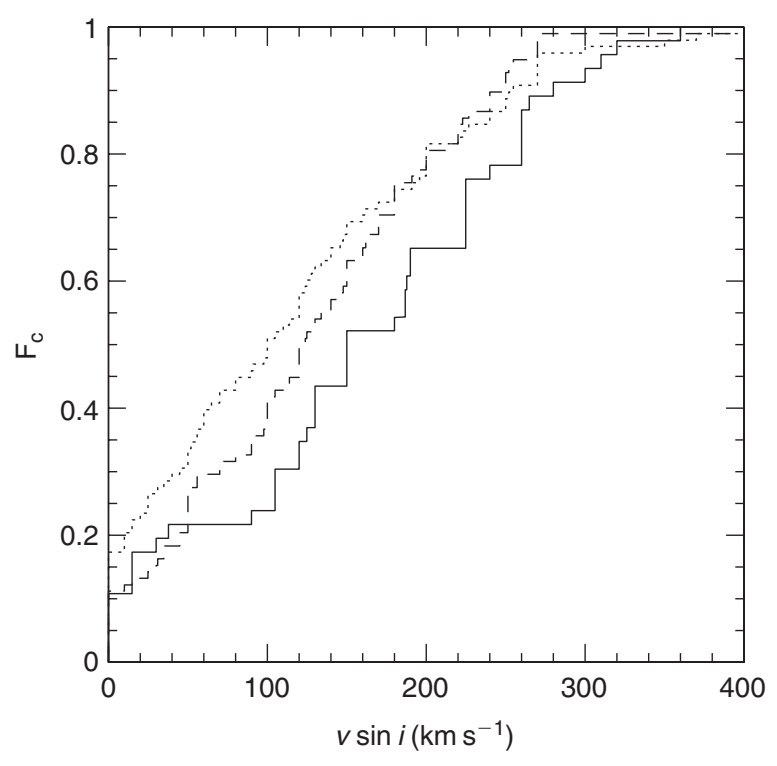

Figure 7 The distribution of projected rotational velocities for the Galactic cluster sample (dotted line) and for the LMC cluster sample (solid line). The dashed line is the distribution for the Galactic cluster sample, after simulation of the systematic effects of low $\mathrm{S} / \mathrm{N}$ (see text).

The resulting KS test between the LMC cluster sample and the $\mathrm{S} / \mathrm{N}$-degraded Galactic cluster sample rates the probability of these two samples been alike at $6 \%$ $\left(D^{+}=0.22, N_{1}=49, N_{2}=151\right)$. This suggests that the average rotation rate is higher amongst the LMC cluster stars. Table 4 summarises our results.

\section{Discussion: The Role of Evolution and Metallicity}

Our observations present us with two important conclusions: 1) Young cluster stars (B0-2 V-III within 2 mag of the MS terminus) are faster rotators than a similar luminosity range in the field; 2) LMC cluster stars are faster rotators than Galactic cluster stars within the same luminosity range, at just under a $2 \sigma$ significance.
Table 4. Mean $v \sin i$ for the four samples discussed in the text

\begin{tabular}{lc}
\hline Location & Mean $v \sin i\left[\mathrm{~km} \mathrm{~s}^{-1}\right]$ \\
\hline Galactic field & 85 \\
Galactic young clusters & 116 \\
LMC field & 112 \\
LMC young clusters & 146 \\
\hline
\end{tabular}

To account, firstly, for the observed difference in rotational velocity between the cluster and field populations, I propose a scenario of evolutionary rotational enhancement. This scenario utilises the one clear difference between the cluster and field populations, that is the spread of age within each population.

Consider a spin-up phase which occurs over an interval of the MS lifetime towards the end of the MS. The small spread of age in the cluster population at the luminosity of the cluster MS terminus places the majority of these stars within the interval of spin-up. The field population, on the other hand, possesses a more uniform spread of ages at a given luminosity, from the ZAMS to the MS terminus (this can be confidently said for such rapidly evolving stars). Hence in the field we see the time-average of the MS rotational velocity.

The evolution of rotational velocity has been examined by Endal \& Sofia (1979) and more recently, by Heger et al. (2000) and Meynet \& Maeder (2000). These models explicitly follow the radial exchange of angular momentum during the course of stellar evolution.

The studies of Heger et al. and Meynet \& Maeder focus on stars of $\geq 20 \mathrm{M}_{\odot}$ which is more massive than that considered in our sample $\left(5-12 \mathrm{M}_{\odot}\right.$; Bressan et al. 1993). A major distinction between massive stars and those considered in our sample is the dominance of mass loss on the course of MS evolution. Their high massloss rates lead to the removal of large amounts of angular 
momentum. Mass loss is enhanced amongst fast rotators that approach the critical angular velocity. The removal of large amounts of mass from $\mathrm{a} \geq 20 \mathrm{M}_{\odot}$ star results in a decline in the angular velocity over the course of MS life.

The study of Endal \& Sofia (1979) focusses on the rotational-velocity evolution of a $5 \mathrm{M}_{\odot}$ star. Here, the mass-loss rates are much reduced. The authors find that stars commencing their MS lives with relatively slow angular velocities remain slow rotators throughout the course of the MS evolution (although there is a marked increase in angular velocity of all stars during the core contraction phase at the exhaustion of central hydrogen burning, this phase is far too short to account for any observable increase in the number of rapid rotators). By contrast, stars commencing their lives with an angular velocity greater than $60-80 \%$ of the critical angular velocity spin-up towards the critical velocity over a moderate fraction of the MS lifetime.

The largest masses considered here are of the order of $12 \mathrm{M}_{\odot}$. Such stars have mass-loss rates 1.5 dex lower than those of a $20 \mathrm{M}_{\odot}$ star (de Jager, Nieuwenhuijzen, \& van der Hucht 1988) and consequently the MS evolution is not dominated by mass loss. Hence, we can assume that the behaviour of the present sample is best described by the models of Endal \& Sofia.

In a cluster, therefore, those stars closer to the MS turnoff are more likely to be rapid rotators, since a proportion of them will have been spun up by evolution. Less-luminous stars that have not evolved as far through the MS phase will not have entered the spin-up phase. This process could provide the mechanism required to explain the more rapidly rotating sample seen in the cluster population in the vicinity of the MS turnoff.

Our second finding, that the LMC cluster and field samples exhibit more rapid rotation than their Galactic counterparts, indicates a metallicity effect. Our sample is insufficient to determine whether the metallicity dependence resides in the initial distribution of rotational velocities or if the magnitude of the evolutionary spin-up discussed above increases in lower metallicity environments. Our observational sample is necessarily limited by the small sample size attainable with our instrumentation. To verify the findings presented here we await the extension to a larger LMC (and SMC) sample. This is currently underway, utilising the FLAMES multi-object spectrograph on the VLT.

\section{Summary}

In this paper I have presented a comparison of the rotational velocities of a sample of stars within the field and within a selection of young clusters in both the Galaxy and, from our own observations, the LMC. I have presented the velocity distribution of 100 LMC early B-type main-sequence stars. This represents the first study of the distribution of extragalactic stellar rotational velocities.

It is found that the early B-star population of young clusters $\left(1-3 \times 10^{7} \mathrm{yr}\right)$ in both the Galaxy and the LMC exhibit more rapid rotation than the field population. I propose this can be explained by a scenario of evolutionary enhancement of the surface angular momentum, brought about by angular momentum redistribution over the mainsequence lifetime.

A comparison of a sample of field and cluster stars, drawn from both LMC and Galactic environments, shows that the LMC stars are more rapid rotators than their Galactic counterparts. The origin of this metallicity dependence is as yet unknown.

\section{References}

Abt, H. A., \& Hunter, J. H. 1962, ApJ, 136, 381

Bernacca, P., \& Perinotto, M. 1971, CoAsi, 239,

Bressan, A., Fagotto, F., Bertelli, G., \& Chiosi, C. 1993, A\&AS, 100,647

Brown, A. G., \& Verschueren, W. 1997, A\&A, 295, 63

Burki, G., \& Maeder, A. 1977, A\&A, 57, 401

Endal, A. S., \& Sofia, S. 1979, ApJ, 232, 531

Gies, D. R., \& Lambert, D. L. 1992, ApJ, 387, 673

González Delgado, R. M., \& Leitherer, C. 1999, ApJS, 125, 479

Gray, D. 1976, The Observation and Analysis of Stellar Photospheres (New York: Wiley)

Guthrie, B. N. G. 1984, MNRAS, 210, 159

Heger, A., Langer, M., \& Woosley, S. E. 2000, ApJ, 528, 368

Hoffeit, D., \& Jaschele, C. 1982, The Bright Star Catalog, 4th ed (New Haven: Yale University Observatory)

Hoffeit, D., Saladyga, M., \& Wlasuk, P. 1988, A Supplement to the Bright Star Catalog (New Haven: Yale University Observatory)

de Jager, C., Nieuwenhuijzen, H., \& van der Hucht, K. A. 1988, A\&AS, 72, 259

Keller, S. C., Wood, P. R., \& Bessell, M. S. 1999, A\&AS, 134, 489 Keller, S. C., Bessell, M. S., \& Da Costa, G. S. 2000, AJ, 119, 1748

Keller, S. C., Bessell, M. S., \& Da Costa, G. S. 2001a, AJ, 121, 905

Keller, S. C., Grebel, E. K., Miller, G. J., \& Yoss, K. M. 2001b, AJ, 122, 248

Keller, S. C., \& Wood, P. R. 2002, ApJ, 578, 144

Korn, A. J., Becker, S. R., Gummersback, C. A., \& Wolf, B. 2000 , A\&A, 353, 655

Korn, A. J., Keller, S. C., Kaufer, A., Langer, N., Przybilla, N., Stahl, O., \& Wolf, B. 2002, A\&A, 385, 143

Lennon, D. J., Dufton, P. L., Mazzali, P. A., Pasian, F., \& Marconi, G. 1996, A\&A, 314, 243

McErlean, N. D., Lennon, D. J., \& Dufton, P. L. 1999, A\&A, 349, 553

Maeder, A., Grebel, E. K., \& Mermilliod, J.-C. 1999, A\&A, 346, 459

Mermilliod, J.-C. 2000, in ASP Conf. Ser. 198, Stellar Clusters and Associations: Convection, Rotation, and Dynamos, eds. R. Pallavicini, G. Micela, \& S. Sciortino (San Francisco: ASP), 513

Meynet, G., \& Maeder, A. 2000, A\&A, 361, 101

Robertson, J. W. 1974, ApJ, 191, 67

Rolleston, W. R. J., Brown, P. J. F., Dufton, P. L., \& Howarth, I. D. 1996, A\&A, 315, 95

Royer, P. 2003, in Proceedings of the IAU Symposium 215 on Stellar Rotation, eds. P. Eenens, \& A. Maeder

Slettebak, A. 1968, ApJ, 154, 933

Slettebak, A., Collins, G. W., Parkinson, T. D., Boyce, P. B., \& White, N. M. 1975, ApJS, 29, 137

Townsend, R. H. D., Owocki, S. P., \& Howarth, I. D. 2004, MNRAS, submitted (astro-ph:0312113)

Venn, K. A. 1995, ApJ, 449, 839

Venn, K. A. 1999, ApJ, 518, 405

von Zeipel, H. 1924, MNRAS, 84, 665

Wolff, S. C., Edwards, S., \& Preston, G. W. 1982, ApJ, 252, 322

Zorec, J., \& Briot, D. 1991, A\&A, 245, 150

Zorec, J., \& Briot, D. 1997, A\&A, 318, 443 